\title{
Automated pathological growths parametrization based on computed tomography layer segmentation
}

\author{
N.I. Limanova ${ }^{1}$, S.G. Ataev ${ }^{1}$ \\ ${ }^{I}$ Volga State University of Telecommunications and Informatics, Lev Tolstoy str. 23, 443010, Samara, Russia
}

\begin{abstract}
Nowadays the multi-layer computed tomography (CT) shots with contrast dye used for detection of small pathological growths regions are widespread in medical clinics. Although, CT study with contrast dye use has contraindications and is much more expensive for patients than study without use of dye. This article proposes the algorithm based on segmentation of multi-layer CT (without dye use). The algorithm allows to automatically identify pathological growths and to detect studying object regions. Algorithm also evaluates parameters of small objects and sinuses, which occupy only small part of initial CT layers, with high accuracy.
\end{abstract}

Keywords: Image analysis; computed tomography; computer-aided diagnosis; parameterization; software development

\section{Introduction}

X-ray computer tomography (CT) was developed in the 1970s and mass adopted in 1980s. Now CT is widely used for meeting different diagnostic needs in outpatient and inpatient medical care. Accessibility of CT scan devices led to the development of computer-aided diagnosis (CAD) systems, whose task is to increase the accuracy and speed of diagnosis process. CAD systems provide medics an additional data extracted from CT scans. Currently, medics are just beginning to use CADs in their everyday medical practice, but CAD development fundamentals is actively developing field of research [1,2]. Some cases require to evaluate numerical parameters of small anatomical structures (such as measuring the volume of maxillary sinus and examining it if there are the pathological growths). This article proposes the algorithm that allows to calculate automatically the parameters of CT scan objects, including sinuses and small structures taking a small part of the picture.

The development of multi-layer CT has led to increased speed and effectiveness of medical examination. Array of multi-layer CT layers represents comprehensive information about inner structure of the examining object. Nowadays, there is a lot of CT visualization improvement $[3,4]$ and 3D-reconstruction methods are developed $[5,6,7]$. They can simplify CT scan reading process, but can't lead to automatic parameter evaluation. Also, there are some methods that analyze CT layers separately, for example pulmonary nodes classification methods $[8,9,10]$. This kind of methods is suitable for object classification problems, which can be solved with the use of neural networks [11]. All these methods can't be used for automated parameterization of small anatomic structures situated in small part of CT layers.

Nowadays the CT study with the use of contrast dye is widely used for detection of small pathological growths areas. The CT of maxillary sinus with contrasting should be performed after studying without contrasting. The purpose of this additional study is the differentiation of the maxillary sinus growth (such as cyst, polypoid growths, etc.). Contrast dye accumulates in tumor cells and marks them at CT scan layers, as a result soft tissues become distinctly visible. However, the CT study with use of contrast has contraindications. It is significantly more expensive and requires additional CT session. The article proposes CT scan segmentation algorithm that implements detection of the anatomic structure area and allows automatic detection of the pathological growths on CT scans, which are taken without contrast dye use.

\section{The structure of multi-layer CT scan}

A multi-layer CT scan structure gives a possibility for medics to receive comprehensive information about inner structure of scanned objects. Thickness of a single layer can vary (within tomograph capabilities) and is selected depending on specifics of the studied organs. Distinctive feature of multi-layer CT is placement of the studied objects on several layers at once. Each layer of a tomogram projection gives exact value of tissue density displayed by the corresponding pixels in a picture. The pixels have gradation of color from light to dark shades of gray. The gray shade is lighter, the tissue within pixel is denser. Thus, corresponding to studying object pixel set contains an object structure comprehensive data and allows its automated analysis and study [12].

Because of low brightness resolution of monitors that can't represent whole range of possible densities, every CT scan has its own density range called "Hounsfield scale". Density of any tissues shown on CT layer is measured in Hounsfield units. This range is selected depending on type of studying object. The tissues inside the "Hounsfield scale" are represented by shades of grey, which brightness is in direct ratio of the tissue density, while tissues outside the range are representing by black or white color (less than minimum or more than maximum, respectively). This range is set by a two numbers - center and width. CT scan layer example is shown on fig. 1. Values W: 90 and C: 40 (Hounsfield units) are width and center of Hounsfield scale. 


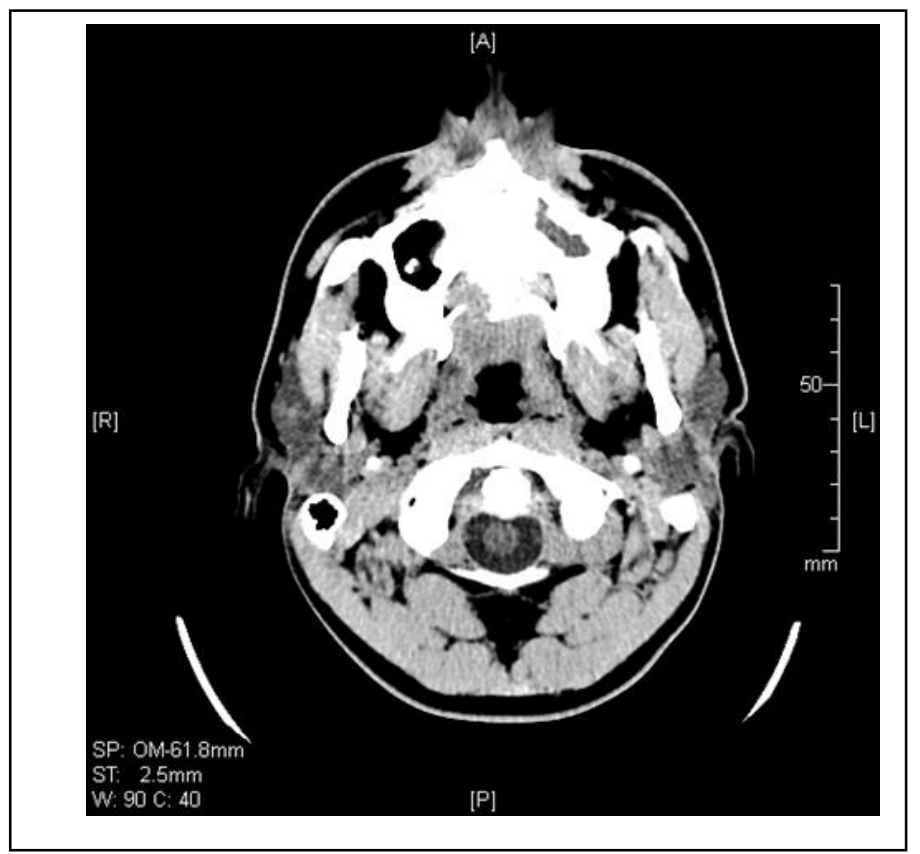

Fig. 1. CT layer example, which contains Hounsfield Scale information.

\section{The algorithm for automated small object parameterization}

Consider the proposed automated object parameterization algorithm and the exemplary problem of maxillary sinus study. Task of automated object parametrization has been divided into two subtasks:

- CT layers segmentation (finding an object region and corresponding subset of pixels related to this object);

- Object pixels analysis (evaluating required parameter values of the object of study).

Proposed CT layer segmentation algorithm starts from receiving from user a set of CT layers. Then user should specify starting point of studying object search and set the criterion value, required for checking the pixels belonging to this object. This criterion applies as a threshold value in further algorithm performance and can be specified as a brightness value for layer pixels in range of 0 (black) to 1 (white) or as density value for tissues (in Hounsfield Units). Fig. 2 represents developed software graphic user interface with loaded set of CT layers.

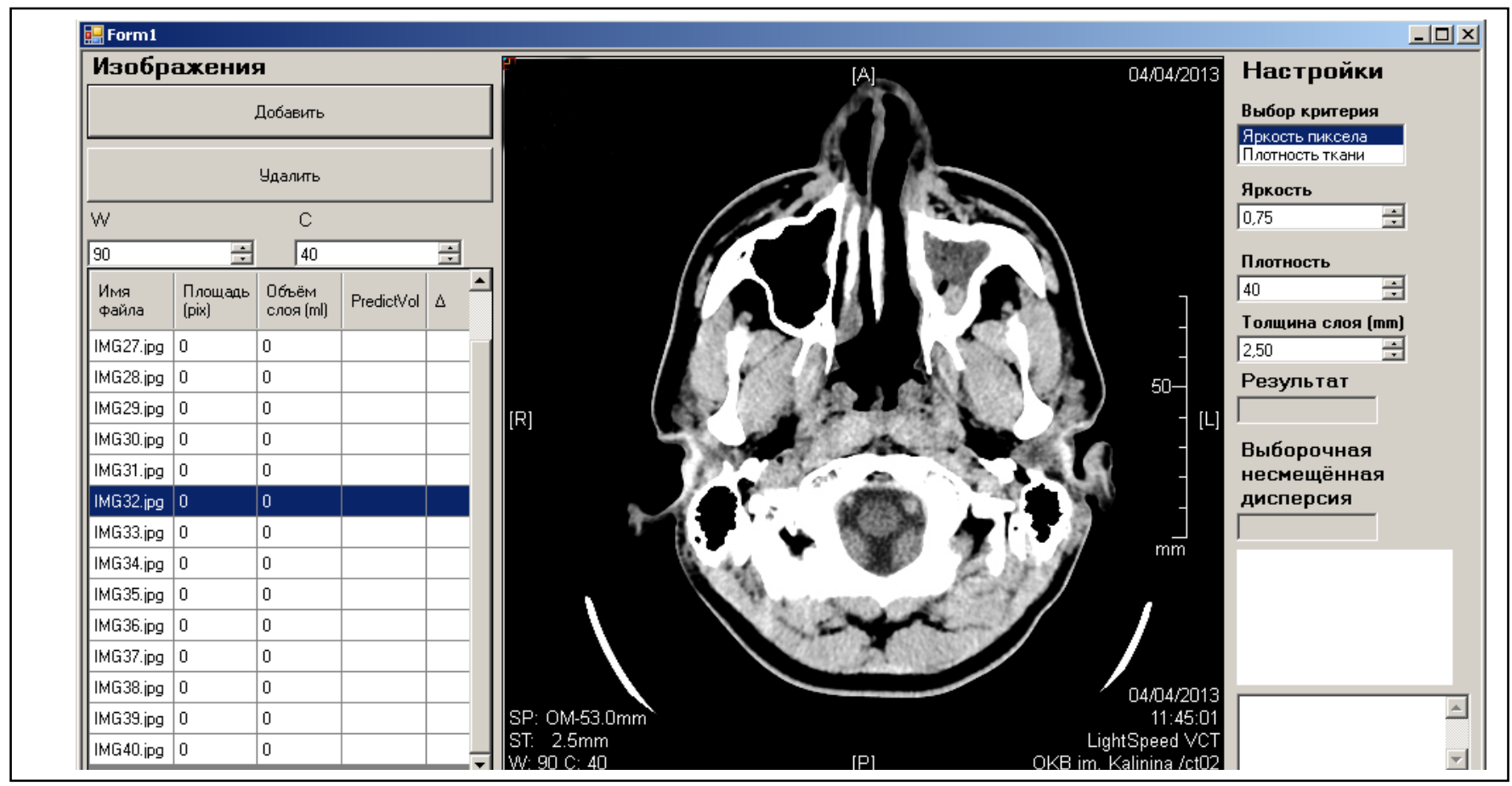

Fig. 2. Developed software graphic user interface with CT layers loaded

At first, object related pixel subset contains only one starting pixel specified by user by clicking on the CT layer. Then every other pixel adjacent to object region is compared with object belonging criterion. Pixel is added to object related pixel set if it fits the criterion, otherwise it is marked as an object region border pixel. These pixels don't extend the object related region, but they can be useful later. 
Image Processing, Geoinformation Technology and Information Security / N.I. Limanova, S.G. Ataev

In case the object border on a separate layer of a picture isn't closed, and it needs to be completed, the algorithm performs the following operations. Set of existing border pixels allows doing an object border restoration. Although algorithm puts a restriction for the starting layer to have a closed borderline. Pixel search process on the starting layer ends when all object's adjacent pixels are checked and there is no possibility to extend studying object region, then algorithm performs possible extensions of object region to adjacent layers while it's possible. Every new layer should be processed the same way as first layer, but starting pixel set is defined by object region on previous layer. If adjacent layer hasn't got any pixels that fit the criterion, region extension in this direction stops. Segmentation algorithm completes when there are no further possibilities for region extension.

CT layers without closed object region borderline require additional borderline finding, which allows to strictly identify which part of layer is refers to studying object. Before examining next layer, algorithm performs extrapolation for area value prediction. If current region area significantly exceeds predicted value, it's borderline got unclosed character and region extension to this layer cancels. Set of pixels referring to external borderline has a shape of curved line. Algorithm searches for center of borderline, which divides its pixels to two subsets, then checks all pixel combinations from first and second subset. Borderline closes with line built through pair of points, which have highest w value, which shall be determined from the following equation:

$$
w=\frac{l^{\alpha}}{d^{\beta}}
$$

where $l$-length of borderline piece between two points, $d$-distance between those points.

$\alpha$ и $\beta$ rates are needed to receive a few possible optional border closings and to choose the one that leads to area value which better fits the prediction. Fig. 3 shows fragment of initial CT layer (left) and borderline detection and closing (right). Squares mark the center of borderline and points, which has been chosen for borderline closing.

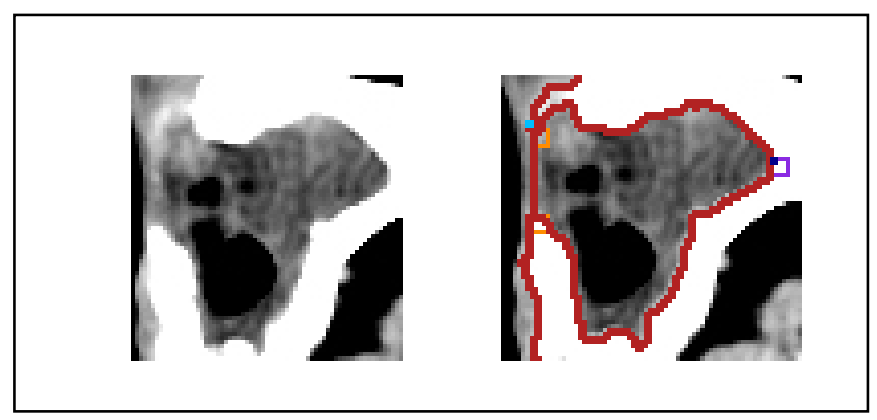

Fig. 3. CT layer example (left) with automated borderline closing (right).

\section{Results}

Result of described CT layer segmentation algorithm is set of pixels related to studying object, which allows to do an analysis of this object and evaluate its parameters, such as volume, density distribution, density variance, layer areas and layer volumes. Software realization of developed algorithm allows browsing CT layers with marked object area and provides density distribution histogram. Studying object histogram can be used to identify its inner growths amount and character. Therefore it allows checking the possible pathological character of those growths.

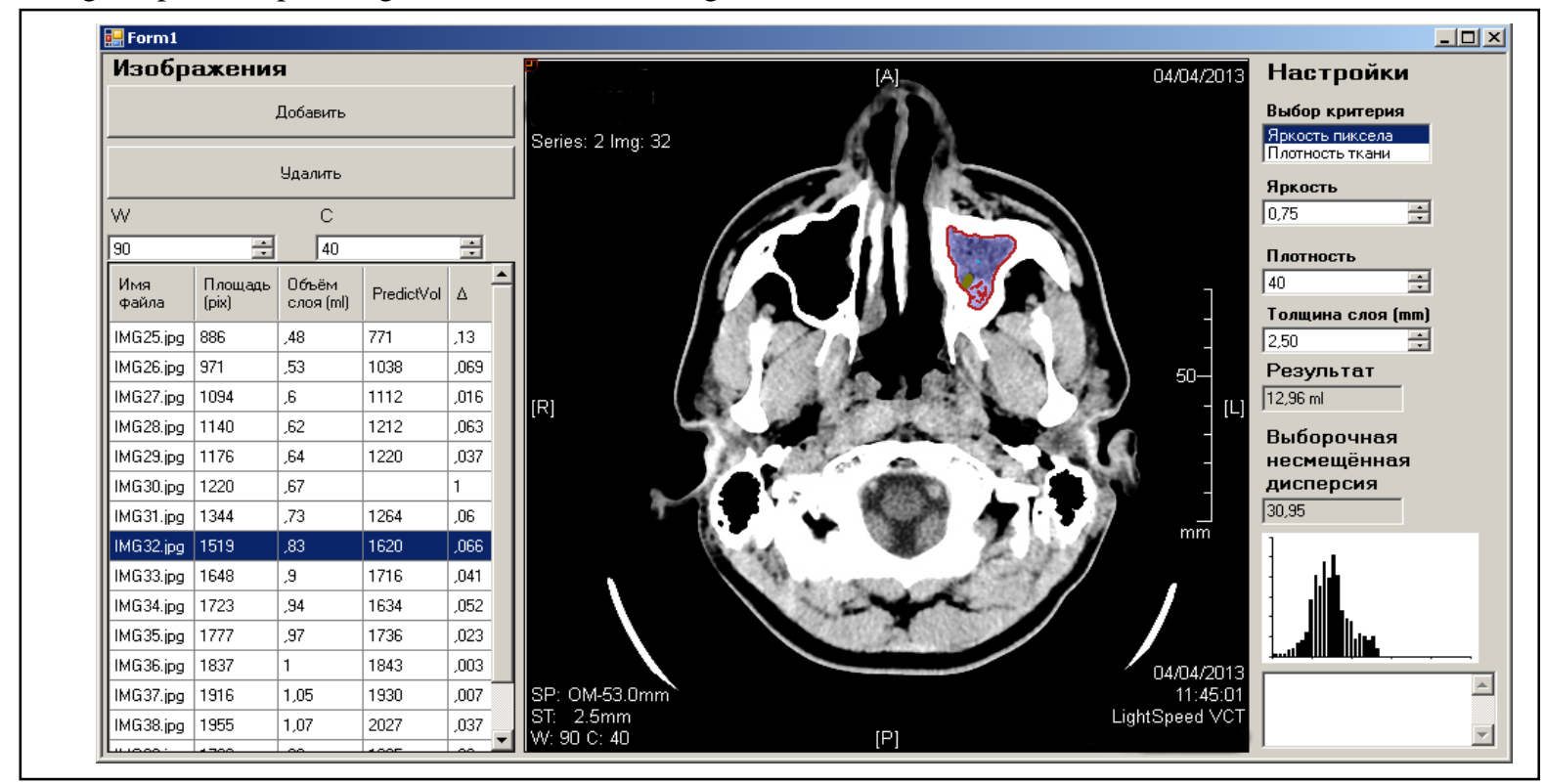

Fig. 4. Developed software user interface with object parameterization results.

Maxillary sinus study with application of developed algorithm has led to following results. Maxillary sinus total volume has been calculated as sum of its volume on each CT layer, and volume for each layer is product of object area value and layer 
Image Processing, Geoinformation Technology and Information Security / N.I. Limanova, S.G. Ataev

thickness. The volume value for studied maxillary sinus equals $12,96 \mathrm{ml}$, and fig. 4 represents its analysis results. Developed algorithm and software application allows possible pathological growths visualization without use of contrast dye.

For example, fig. 4 shows CT layer with studied object area, with dark green color for object part which filled with air and shades of blue for tissues with density higher than density of air (which fills healthy maxillary sinus). Initial CT layers contain density information that allows doing this division after each layer segmentation. This kind of tissue localization can be alternative to contrast dye use.

Fig. 5 shows density distribution histograms of two maxillary sinuses. Left one mainly contains air, you can clearly see it by 0.92 value of its histogram left column, which corresponds to density less than lower boundary of Hounsfield range (i.e. air). Right one contains big amount of possibly pathological growths, so its histogram shows the densities distribution of those growths. Variance value of density can be used as distribution heterogeneity mark. For right maxillary sinus (marked at fig. 4) this value equals 30.95 , while left one's equals 8.01 . With a density distribution histogram, it illustrates heterogeneity degree of inner growths and demonstrates existence possibility of pathological growths.

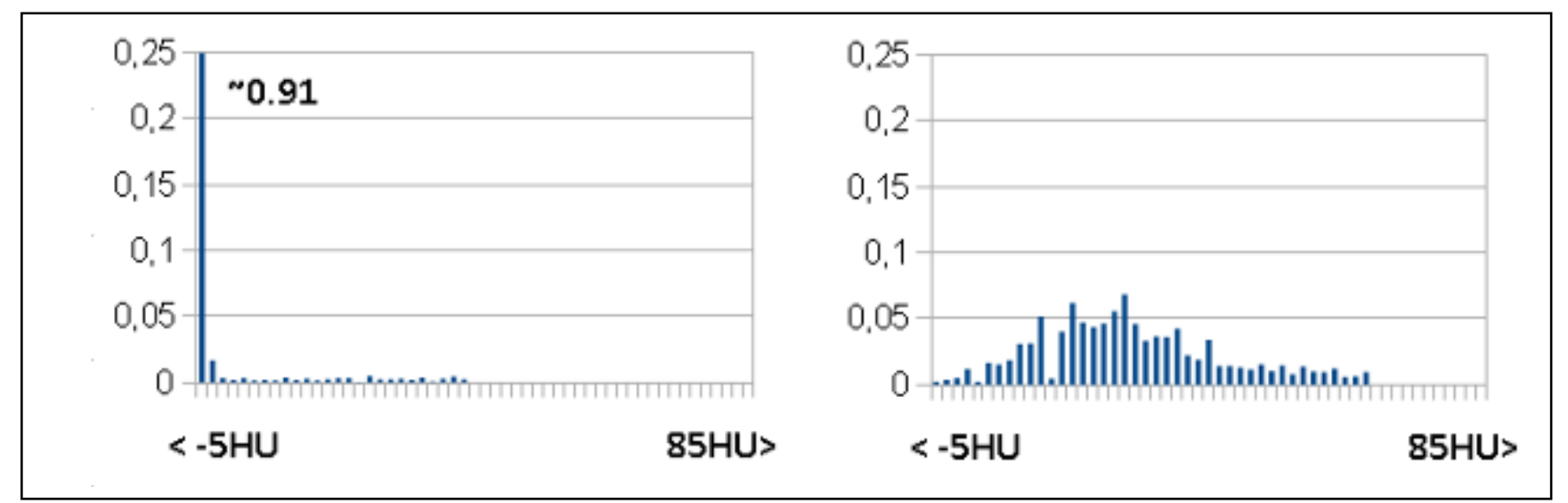

Fig. 5. Density distribution histogram of maxillary sinus (left - maxillary sinus with normal anatomical structure, filled with air, right - maxillary sinus with possible pathological growths).

\section{Conclusion}

Developed algorithm application leads to multi-layer CT automated analysis through object parameters calculation and region visualization. Additional region division allows finding specific types of tissues, detecting possible pathological tissue regions (the same way as it happens with contrast dye use). Density distribution histogram shows inner growth properties of studying object. Density distribution variation indicates inhomogeneity grade of its inner growth.

Automated parameters evaluation allows extending diagnostic data available to medics. Developed software passed approbation in the V.D. Seredavin Samara regional clinic and helps to improve diagnosis study of CT shots without use of contrast dye.

\section{References}

[1] Van Ginneken B, Schaefer-Prokop CM, Prokop M. Computer-aided diagnosis: how to move from the laboratory to the clinic. Radiology 2011; 261(3): 719732.

[2] Doi K. Current status and Future Potential of Computer-Aided Diagnosis in Medical Imaging. The British Journal of Radiology, 2005 ; 78 : 3-19. DOI: $10.1259 / \mathrm{bjr} / 82933343$.

[3] Seletchi E, Duliu O. Image Processing and Data Analysis in Computed Tomography. Romanian Journal of Physics, 2007; 1: 764-774. URL: http://www.nipne.ro/rjp/2007_52_5-6/0667_0667.pdf (19.06.2015).

[4] Boussion N, Fayad H, Le Pogam A, Pradier O, Visyikis D. Image Processing Methods in CT for Radiotherapy Applications. Theory and Applications of CT Imaging and Analysis. InTech 2011: 127-142. URL: http://cdn.intechopen.com/pdfs-wm/14772.pdf (20.06, 2015).

[5] Maher M, Kalra M, Sahani D, Perumpillichira J, Rizzo S, Saini S, Mueller P. Techniques, Clinical Applications and Limitations of 3D Reconstruction in CT of the Abdomen . Korean Journal of Radiology 2004; 5(1): 55-67. DOI: 10.3348/kjr.2004.5.1.55.

[6] Kim HC, Park SH, Park HC, Shin HC, Park SJ, Kim HH, Kim YT, Bae WK, Kim IY. Three-dimensional reconstructed images using multidetector computed tomography in evaluation of the biliary tract. Abdominal Imaging 2014; 29(4): 472-478. DOI: 10.1007/s00261-003-0123-x.

[7] Xu F, Mueller K. Real-time 3D computed tomographic reconstruction using commodity graphics hardware. Physics in Medicine and Biology 2007; 52(12): 3405-3419. DOI:10.1088/0031-9155/52/12/006.

[8] El-Baz A, Beache G, Gimel'farb G, Suzuki K, Okada K, Elnakib A, Soliman A, Abdollahi B. /Computer-aided diagnosis systems for lung cancer: challenges and methodologies. International Journal of Biomedical Imaging 2013; 2013: 1-46. DOI: 10.1155/2013/942353.

[9] Chen H, Xu Y, Ma Y, Ma B. Neural network ensemble-based computer-aided diagnosis for differentation of lung nodules on CT images: Clinical evaluation. Academic Radiology 2010; 17(5): 595-602. DOI: 10.1016/j.acra.2009.12.009.

[10] Montejo L, Jia J, Kim H, Netz U, Blaschke S, Müller G, Hielscher A. Computer-aided diagnosis of rheumatoid arthritis with optical tomography. Part1: feature extraction. Journal of Biomedical Optics 2013; 18(7): 123-137. DOI: 10.1117/1.JBO.18.7.076001.

[11] Chen H, Wang XH, Ma DQ, Ma BR. Neural network-based computer-aided diagnosis in distinguishing malignant from benign solitary pulmonary nodules by computed tomography. Chinese Medical Journal 2007; 120(14): 1211-1215. URL: http://124.205.33.103:81/ch/reader/create_pdf.aspx?file_no= 200771851284230\&year_id=2007\&quarter_id=14\&falg=1 (20.06.2015).

[12] Hofer M. CT Teaching manual: a systematic approach to CT reading. Thieme 2007: 24-25. 